\title{
Measurement of Propagation Effects for High-Speed, Digital UHF Channels
}

\author{
Kent Chamberlin*, Dept. of ECE, Univ. of New Hampshire, \\ kent.chamberlin@unh.edu \\ Andrew Kun, Dept. of ECE, Univ. of New Hampshire, \\ andrew.kun@unh.edu \\ Scott Valcourt, Dept. of Computer Science, Univ. of New Hampshire, \\ sav@unh.edu \\ Benjamin McMahon, Dept. of ECE, Univ. of New Hampshire, \\ benjamin.mcmahon@unh.edu
}

\begin{abstract}
The work described here relates to an effort to assess the reliability and robustness of datacasting for use in the mobile environment. Datacasting employs the excess bandwidth from digital television signals for use in one-way data transmission and it is being used successfully for high-speed downloads at fixed receiver locations. It is being considered as a means for providing data-rich information to police and emergency personnel in the field, and the work reported here is part of the evaluation process.

Datacasting operates in the UHF television band and employs 8-Level Vestigial Sideband (8-VSB) modulation to provide data rates in excess of $2.5 \mathrm{Mb} / \mathrm{sec}$. Datacast reception is known to be very vulnerable to multipath interference, particularly time-varying multipath interference. Because of this, an estimation of coverage area based upon signal strength alone does not accurately predict where the signal will be available. While propagation models are available that can predict both signal strength and multipath, they cannot provide the type of statistical information about signal availability and reliability that is sought in this study. In order to get the desired information, datacasting receivers and data logging equipment were installed in police vehicles to measure the channel over a wide range of operating conditions. This paper reports on some of the key findings from that study.
\end{abstract}

\section{Introduction}

Datacasting is the term used to describe the transmission of data that can be encoded into the excess bandwidth from digital television signals. Bandwidth is left over when the signal being transmitted does not occupy the entire allotted bandwidth for the channel, and this generally occurs when the television image does not contain a high degree of motion. Data carried via datacasting can be encrypted and sent only to targeted receivers, making this form of data transmission desirable for a range of commercial and government applications.

Datacasting is being used increasingly for one-way, point-to-point data transmission to fixed locations; the work reported here involves the practical considerations associated with using datacasting in mobile environments, and in particular emergency and police vehicles. The mobile datacast evaluation effort described in this paper is currently ongoing and is being carried out in the state of New Hampshire. The datacast signal for the study is provided by New Hampshire Public Television's Channel 57 (731 MHz). Data have been and will be collected over different terrain types (New Hampshire varies from relatively flat, sea-level terrain to rugged mountains containing the highest peak in the 
Northeast) and different weather conditions (data collection will span the summer, fall and winter seasons).

At the time of this writing, datacast and logging equipment have been installed in 9 State Police vehicles, and this paper reports on the findings obtained from those vehicles. As the effort continues, the equipment will be installed in a total of at least 20 cruisers. The objective is to collect a large body of data from these vehicles (position and signal data are logged every second) over the duration of the study so that subsequent data mining can be performed to provide statistically significant assessments of channel reliability. Expectations are that these data will be made available to those wishing to validate channel models.

Due to its sensitivity to multipath interference, particularly time-varying multipath, datacast reception using the current 8-VSB modulation scheme is only practical when the vehicle is not in motion. This may be the greatest limitation associated with using datacasting in the mobile environment. However, this limitation may be mitigated if the proposed Advanced-Vestigial Side-Band (A-VSB) [1] modulation scheme is adopted. The additional error correction added to the data stream with A-VSB will purportedly enable reception while in motion, albeit at a reduction in the data rate.

One surprising finding of this study is that heavy rainfall has been shown to severely degrade datacast reception. An overview of this finding is given in a subsequent section of this paper.

\section{Measured Coverage Area}

One major result of the data logging effort is the generation of maps showing where datacast reception is possible. Figure 1 shows datacast coverage for most of the state of New Hampshire along with a computer-model generated coverage map for the television transmitter. The measured coverage is represented by colored dots corresponding to locations where a vehicle stopped (since the receiver can only lock when stationary) and the receiver either did or did not lock to the datacast signal. Green dots represent locations where lock occurred with a high-average signal-to-noise ratio (SNR), yellow for a lock with marginal average SNR and red for no lock. As expected, the location of data points corresponds to the roadways patrolled by the cruisers.
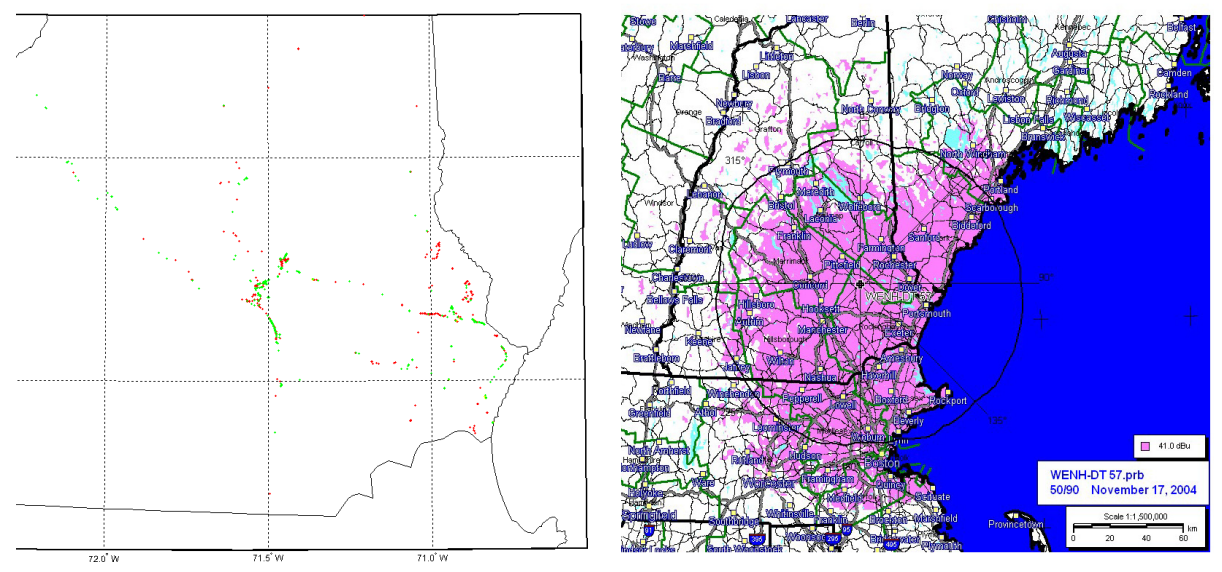

Figure 1. Measured Coverage Area for Datacasting on the Left and Modeled Television Coverage Area on the Right.

The signal-to-noise ratio values used in generating the coverage maps often come from measurements over time by a single vehicle, and/or from multiple vehicles collecting data at the same location (within the 11 meter resolution of the GPS equipment used in this study). Consequently, each dot on the map may represent the average of many individual 
measurements. As this study continues, and more data becomes available, the statistical likelihood of reception at many locations can be calculated.

Referring to Figure 1, it is evident that the measured coverage area for mobile datacast users is generally less than the predicted coverage area for television viewers. There are two fundamental reasons why mobile datacast coverage using low-cost equipment will be less than stationary television coverage. The first reason is that stationary reception is generally obtained using directional antennas with gains of 5-15 $\mathrm{dBi}$ which are elevated in order to provide line of sight propagation paths. The non-directional datacast antennas elevated at trunk height are less conducive to good reception.

The measured signal data are also being plotted on satellite overlays to provide detailed information about where the datacast signal can be received. For example, Figure 2 shows signal reception around Concord, NH which is a challenging reception area because of multiple diffractive terrain features between Concord and the transmitter site.

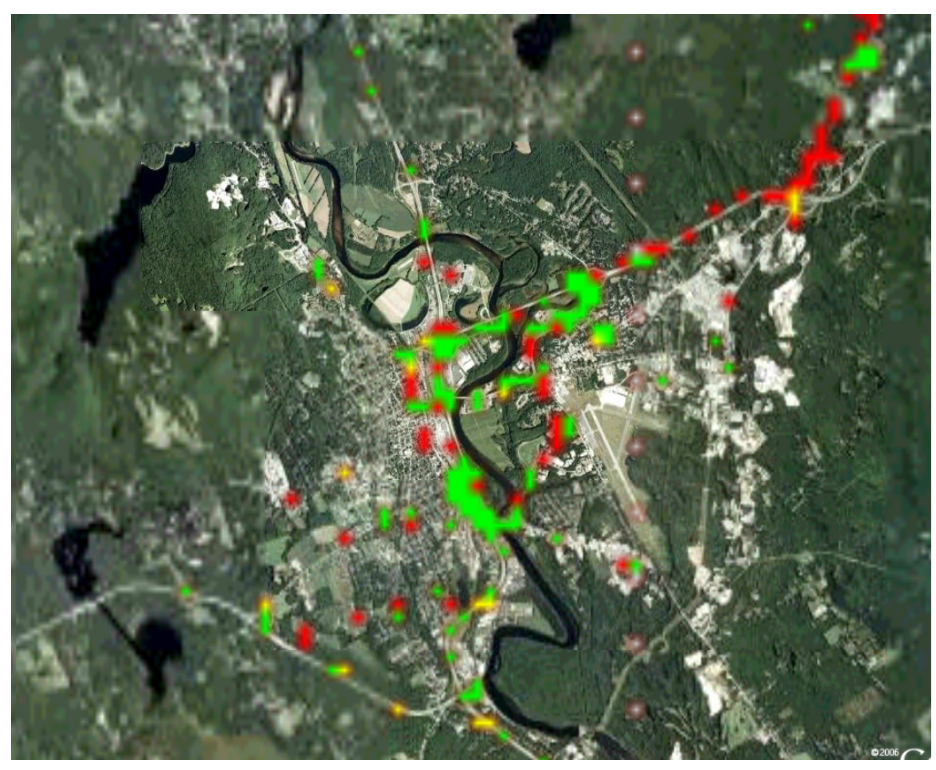

Figure 2. Datacast Coverage Measured in the Vicinity of Concord, NH Shown with a Satellite Overlay.

\section{Rain Effects on Datacast Reception}

While conventional wisdom suggests that short-range, UHF signals will not be impacted by rainfall, there were several documented occurrences where datacast transmission outages coincided with heavy rainfall. Because the overall objective of this study is to investigate the capabilities and limitations of the datacast system, gaining an understanding of the rain-related outages has been considered to be a priority.

In response to concern about rain effects, a fixed datacast monitor was installed to enable rain effects to be documented explicitly. Key parameters defining datacast signal robustness (e.g., signal strength and signal-to-noise ratio) are recorded every second, and radar display information along the propagation path is being recorded every five minutes.

An example of a datacast rain degradation event is seen in Figure 3, which was collected during a heavy thunderstorm in July 2006. The red color code on the radar image corresponds to an equivalent reflectivity of $50 \mathrm{dBZ}$, which corresponds to a rainfall rate of at least 1.75 inches per hour. It is seen in the corresponding plot of SNR that the SNR decreases by at least $20 \mathrm{~dB}$ as the easterlytraveling storm passes between the transmitter and monitor. This dip in SNR 
would be sufficient to render the datacast signal essentially unusable for most of the normal coverage area.
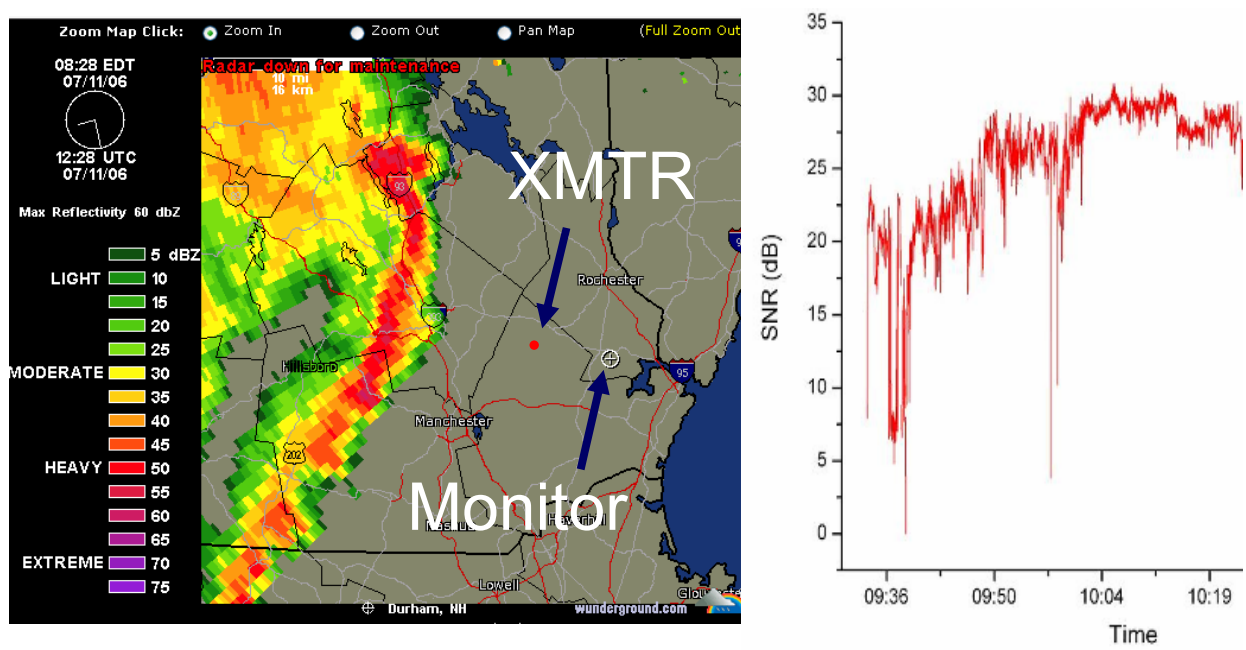

Figure 3. Weather Radar Image and Received Signal-to-Noise Ratio Collected on July 11, 2006. The Storm is Moving from West to East.

During the observed outages, the signal strength remained at above-reception levels while the signal-to-noise ratio dropped below the reception threshold. This suggests that that the outages are not caused by signal attenuation, which is to be expected given the low rain-attenuation rates for datacast frequencies. The assumed cause of the outages is the Doppler shift and/or multipath interference created by regions of intense and moving rainfall. From the observations made to date, only rainfall amounts in excess of around $50 \mathrm{dBZ}$ will create outages, while lesser amounts do not appear to measurably affect system performance.

\section{Conclusions}

The datacast signal can provide high-speed downloads to stationary vehicles. Using an antenna, preamplifier and receiver with a total cost of around $\$ 300$, the datacast transmission can be received within a reasonable portion of a digital television transmitter's coverage area. The 8-VSB is susceptible to multipath interference from terrain traffic and heavy rainfall, making it impractical to predict channel performance with existing propagation models.

Additional measurements will be necessary to fully gage the suitability of this channel for its intended purpose.

\section{Reference}

[1] Glen Dickson, "Mobile DTV Gets Its Road Test”, Broadcasting \& Cable Magazine, November 6, 2006 